\title{
artigo
}

\section{Diagnósticos e intervenções de enfermagem em idosos frágeis segundo o modelo conceitual de henderson}

\author{
Nursing diagnoses and interventions in frail elderly according to henderson's conceptual model \\ Diagnósticos e intervenciones de enfermería en ancianos frágiles según el modelo conceptual de henderson
}

\begin{abstract}
RESUMO
Objetivo: Identificar os principais Diagnósticos de Enfermagem (DE) em idosos frágeis, propondo intervenções segundo o modelo conceitual de Henderson. Método: Trata-se de um estudo transversal, descritivo com abordagem quantitativa e qualitativa, realizado com 25 idosos de uma Instituição de Longa Permanência. Foram aplicados um questionário sociodemográfico e econômico, o Mini Exame do Estado Mental e a escala de fragilidade de Edmonton. Para elaboração dos enunciados de diagnósticos e intervenções de enfermagem, empregou-se a CIPE ${ }^{\circledR}$, versão 2017. Os dados foram processados no SPSS, versão 20 e analisados através da estatística descritiva. Resultados: Os DE mais frequentes foram: Marcha prejudicada $(94,4 \%)$, Risco de queda $(94,4 \%)$, Memória prejudicada (94,4\%), Falta de apetite $(66,7 \%$ ) e Humor deprimido (50\%). Conclusão: A execução do estudo evidenciou que $72 \%$ dos participantes apresentaram fragilidade, onde foi possivel identificar 8 DE dentre os idosos frágeis, possibilitando a formulação de intervenções frente às necessidades de cuidados.
\end{abstract}

DESCRITORES: Diagnóstico de Enfermagem; Idoso; Fragilidade.

\section{ABSTRACT}

Objective: To identify the main Nursing Diagnoses (ND) in frail elderly, proposing interventions according to Henderson's conceptual model. Method: This is a cross-sectional, descriptive study with a quantitative and qualitative approach, conducted with 25 elderly people from a long-term institution. A sociodemographic and economic questionnaire, the Mini Mental State Examination and the Edmonton Frailty Scale were applied. To elaborate the statements of nursing diagnoses and interventions, the ICNP® was used, version 2017. The data were processed in the SPSS, version 20 and analyzed through descriptive statistics. Results: The most frequent ND were: Impaired gait $(94.4 \%)$, Risk of falling $(94.4 \%)$, Impaired memory (94.4\%), Lack of appetite $(66.7 \%)$ and Depressed mood (50\%). Conclusion: The execution of the study showed that $72 \%$ of the participants presented frailty, where it was possible to identify 8 ND among the frail elderly, enabling the formulation of interventions in view of care needs. DESCRIPTORS: Nursing Diagnosis; Elderly; Fragility.

\section{RESUMEN}

Objetivo: Identificar los principales Diagnósticos de Enfermería (DE) en ancianos frágiles, proponiendo intervenciones de acuerdo con el modelo conceptual de Henderson. Método: Se trata de un estudio transversal y descriptivo con un enfoque cuantitativo y cualitativo, realizado con 25 personas mayores de una institución a largo plazo. Se aplicó un cuestionario sociodemográfico y económico, el Mini Examen del Estado Mental y la Escala de Fragilidad de Edmonton. Para elaborar las declaraciones de diagnósticos e intervenciones de enfermería, se utilizó el CIPE ${ }^{\circledR}$, versión 2017. Los datos se procesaron en la versión 20 de SPSS y se analizaron mediante estadísticas descriptivas. Resultados: Los DE más frecuentes fueron: Dificultad para la marcha $(94,4 \%)$, Riesgo de caída $(94,4 \%)$, Deterioro de la memoria $(94,4 \%)$, Falta de apetito $(66,7 \%)$ y estado de ánimo deprimido $(50 \%)$. Conclusión: La ejecución del estudio mostró que el 72\% de los participantes presentaban fragilidad, donde era posible identificar 8 DE entre los ancianos frágiles, permitiendo la formulación de intervenciones en vista de las necesidades de atención.

DESCRIPTORES: Diagnóstico de Enfermería; Ancianos; Fragilidad.

RECEBIDO EM: 27/07/2020 APROVADO EM: 28/07/2020

\section{Vitória Polliany de Oliveira Silva}

Enfermeira, pela Faculdade Internacional da Paraíba (FPB).

ORCID: 0000-0003-1363-6940 


\section{Lucilla Vieira Carneiro}

Enfermeira. Mestre em Enfermagem, pela Universidade Federal da Paraíba (UFPB).

ORCID: 0000-0002-6988-2297

\section{Neyce de Matos Nascimento}

Enfermeira. Mestre em Gerontologia, pela Universidade Federal da Paraíba (UFPB).

ORCID: 0000-0001-5557-3365

\section{INTRODUCTION}

A população brasileira está vivenciando um processo de transição demográfica, decorrente da redução das taxas de mortalidade e natalidade. Tal cenário, vem contribuindo para um contigente significativo de pessoas idosas ${ }^{(1)}$. Segundo projeções, o Brasil será o sexto país do mundo com maior número de idosos e em 2050, eles representarão $18 \%$ do total de habitantes ${ }^{(2)}$.

O envelhecimento traz consigo alterações que interferem diretamente na saúde, proporcionando o comprometimento da capacidade física e mental. Essa realidade, torna os idosos dependentes na realização das atividades de vida diária. Nesses casos, a ausência de cuidador a domicílio e os conflitos familiares, favorecem a demanda por Instituições de Longa Permanência para Idosos (ILPIs), destinadas à moradia coletiva de indivíduos com idade igual ou superior a 60 anos, que possuem ou não suporte familiar e em condições de liberdade, dignidade e cidadania ${ }^{(3)}$.

As ILPIs constituem um desafio à adesão de síndrome da fragilidade entre os residentes, já que as alterações do próprio envelhecimento e as doenças pré-existentes tendem a se agravar mediante as condições impostas nesses ambientes ${ }^{(4)}$.

A fragilidade caracteriza-se como uma condição não ótima dos idosos, de causa multifatorial e dinâmica, decorrente da relação de aspectos de cunho biológico, psicológico e social durante todo o ciclo de $\operatorname{vida}^{(5)}$. Propicia prejuízos funcionais e eventos adversos à saúde e é considerada uma das principais causas de mortalidade precoce e morbidade no público idoso ${ }^{(6)}$. No entanto, pode ser reversível ou retardada, através de avaliação multidisciplinar abrangente e com o auxílio de tratamento adaptado as necessi- dades individuais ${ }^{(7)}$. Sua investigação possibilita a identificação de idosos frágeis e tem sido foco da geriatria e gerontologia ${ }^{(8)}$.

A assistência de enfermagem é fundamental para proporcionar qualidade de vida a esse segmento populacional. Para isso, o Processo de Enfermagem, instrumento metodológico que orienta o cuidado profissional, é visto como uma ferramenta fundamental para subsidiar a assistência prestada pelo enfermeiro aos idosos frágeis. É composto por cinco etapas: Coleta de dados, Diagnóstico de Enfermagem (DE), Planejamento, Implementação e Avaliação. A segunda etapa, relacionada aos DE proporciona o julgamento clínico do indivíduo frente aos problemas de saúde e norteia o desenvolvimento de Intervenções de Enfermagem (IE) $)^{(9)}$, cujas terminologias podem ser encontradas na Classificação Internacional para a Prática de Enfermagem $\left(\mathrm{CIPE}^{\circ}\right)$, a qual permite o desenvolvimento de uma linguagem universal, precisa e objetiva, garantindo a continuidade de cuidados prestados pela equipe de enfermagem ${ }^{(10)}$.

Nesse ínterim, os cuidados de enfermagem devem ser baseados nos saberes teórico-filosóficos da profissão, envolvendo além do uso de terminologias, as teorias de enfermagem. A aplicabilidade da Teoria das Necessidades Humanas Fundamentais proposta por Virginia Henderson, favorece a busca de autonomia e independência entre os idosos fragilizados, sendo de grande relevância para a prática clínica do enfermeiro. Esse modelo teórico abrange quatorze necessidades humanas fundamentais, distribuídas em quatro componentes de cuidado: Biológico/Fisiológico, Psicológico, Social e Espiritual/Moral ${ }^{(11)}$.

O conhecimento das necessidades deste público, possibilita o aprimoramento de uma assistência integral e previne a ocorrência de complicações que agravem a condição de saúde ${ }^{(12)}$. Dessa forma, objetivou-se, identificar os principais diagnósticos de enfermagem (DE) em idosos frágeis, propondo intervenções segundo o modelo conceitual de Henderson.

\section{MÉTODO}

Trata-se de estudo transversal, descritivo com abordagem quantitativa e qualitativa, realizado em uma ILPI, no município de João Pessoa-PB, Brasil.

Para a composição da amostra, os critérios de inclusão foram: ter idade igual ou superior a 60 anos e residir na referida ILPI. Foram excluídos do estudo, os idosos que apresentaram diagnóstico sugestivo de demência conforme resultado obtido no Mini Exame do Estado Mental (MEEM)(13), restritos ao leito ou a cadeira e portadores de comprometimento verbal, visual e auditivo. Dessa forma, a amostra totalizou 25 participantes.

A pesquisa foi realizada no período de agosto de 2019 e foi subsidiada pelos seguintes instrumentos: um questionário sociodemográfico e econômico, o MEEM para avaliar a função cognitiva e a Edmonton Frail Scale (EFS), para avaliação da fragilidade. Essa escala foi traduzida e adaptada transculturalmente para a língua portuguesa. Considerou-se frágil, os idosos com pontuação $\geq$ a sete si4). $^{(14}$.

Após a identificação dos idosos com fragilidade e de acordo com as condições clínicas observadas através das necessidades humanas fundamentais, segundo o modelo conceitual de Henderson, foram elaborados diagnósticos e intervenções de enfermagem utilizando-se a CIPE, versão $2017^{(15)}$.

Os dados foram organizados em uma planilha no Microsoft Office Excel 2016 e 


\section{artigo}

processados no software SPSS, versão 20.0. Para realização da análise, foi utilizada a estatística descritiva, com frequências absolutas e relativas, médias e desvio padrão.

Foram respeitados os preceitos éticos e legais seguidos nas investigações envolvendo seres humanos, em consonância as diretrizes estabelecidas pela Resolução 466/12 do Conselho Nacional de Saúde(16). O estudo obteve aprovação do Comitê de Ética e Pesquisa do Hospital Universitário Lauro Wanderley da Universidade Federal da Paraíba, sob parecer n ${ }^{\circ} 3.449 .338$ e CAAE $n^{\circ} 13458419.6 .0000 .5183$.

\section{RESULTADOS}

Dos 25 idosos incluídos na amostra, sobressaíram os do sexo feminino (56\%), a média de idade foi de 81,44 anos $( \pm 8,12)$, no que diz respeito ao estado civil (44\%) eram solteiros, (52\%) analfabetos e (84\%) apresentavam renda de até um salário mínimo, com média de $\mathrm{R} \$ 1.237,52( \pm \mathrm{R} \$ 583,97)$.

A fragilidade predominou em $72 \%$ dos participantes, sendo possível elaborar $8 \mathrm{DE}$ e $12 \mathrm{IE}$, os quais foram categorizados segundo os componentes do cuidado de enfermagem, como segue demonstrado no Quadro 1.

\section{DISCUSSÃO}

Observou-se que das necessidades humanas fundamentais, apenas 6 se encontravam prejudicadas nos idosos, favorecendo a elaboração de diagnósticos e intervenções de enfermagem. Ademais, o Componente Espiritual/Moral se manteve preservado.

A "Falta de apetite" constituiu um importante DE, capaz de subsidiar o desenvolvimento de intervenções de enfermagem direcionadas a prevenção de desfechos negativos entre os idosos.

Os dados obtidos são similiares a outra pesquisa nacional, onde a perda de apetite se mostrou acentuada entre os idosos frágeis ${ }^{(17)}$. Com isso, é imprescindível o monitoramento do peso e o gerenciamento nutricional entre esse segmento populacional.

O consumo inadequado de alimentos pode favorecer a ocorrência de desnutrição e aumentar o risco de mortalidade na velhice. No Brasil, o risco de morte em idosos por essa causa tem se mostrado crescente, despertando a necessidade da atuação do enfermeiro frente a redução dessa problemática nos idosos com fragilidade ${ }^{(18)}$.
O DE "Incontinência urinária” foi encontrado em 44,4\% dos idosos frágeis. Os estudos apontam que a fragilidade está fortemente ligada a incontinência urinária, sendo a identificação dos fatores associados, crucial para o aprimoramento de políticas públicas direcionadas à saúde do idoso ${ }^{(19)}$.

O gerenciamento da incontinência urinária neste público é fundamental. Nesse ínterim, alguns métodos conversadores podem ser utilizadas pelos enfermeiros no tratamento de tal distúbrio. Dentre as principais estratégias, encontram-se a execução de exercícios físicos, terapia comportamental e modificações no estilo de vida. Além disso, orientações sobre a redução de ingestão de alimentos considerados irritantes vesicais, é capaz de auxiliar no manejo da incontinência urinária ${ }^{(20)}$.

Os idosos com incontinência urinária, afirmaram fazer o uso de fralda descartável geriátrica, favorecendo a elaboração do DE "Risco de infecção". É importante ressaltar, que a incontinência urinária tem sido apontada como um fator de risco para o desenvolvimento de Infecções do Trato Urinário (ITU) na população idosa. Dentre os mecanismos que contribuem para a

Quadro 1. Distribuição dos Diagnósticos e Intervenções de Enfermagem identificados nos idosos da amostra. João Pessoa, PB, 2019.

\begin{tabular}{|c|c|c|}
\hline $\begin{array}{l}\text { NECESSIDADES HUMANAS } \\
\text { FUNDAMENTAIS }\end{array}$ & DIAGNÓSTICOS DE ENFERMAGEM & INTERVENÇÕES DE ENFERMAGEM \\
\hline \multicolumn{3}{|c|}{ COMPONENTE BIOLÓGICO/FISIOLÓGICO } \\
\hline Comer e beber & Falta de apetite $(66,7 \%)$ & $\begin{array}{l}\text { Gerenciar condição nutricional; } \\
\text { Pesar paciente }\end{array}$ \\
\hline Eliminar os resíduos corporais & Incontinência urinária $(44,4 \%)$ & $\begin{array}{l}\text { Gerenciar incontinência urinária; } \\
\text { Orientar sobre manejo da incontinência urinária }\end{array}$ \\
\hline Mover-se e manter uma boa postura & Marcha prejudicada $(94,4 \%)$ & $\begin{array}{l}\text { Auxiliar na marcha; } \\
\text { Monitorar risco de queda; } \\
\text { Orientar sobre prevenção de queda }\end{array}$ \\
\hline $\begin{array}{l}\text { Manter o corpo limpo, cuidado e proteger o } \\
\text { tegumento }\end{array}$ & Risco de queda $(94,4 \%)$ & $\begin{array}{l}\text { Obter dados sobre autocuidado; } \\
\text { Prevenir infecção }\end{array}$ \\
\hline \multicolumn{3}{|c|}{ COMPONENTE PSICOLÓGICO } \\
\hline Aprender & Memória prejudicada $(94,4 \%)$ & Promover uso de técnica de memória \\
\hline \multicolumn{3}{|c|}{ COMPONENTE SOCIAL } \\
\hline Ocupar-se com vistas à autorrealização & $\begin{array}{l}\text { Humor deprimido }(50 \%) \\
\text { Falta de apoio social }(11,1 \%)\end{array}$ & $\begin{array}{l}\text { Gerenciar humor; } \\
\text { Promover apoio social }\end{array}$ \\
\hline Fonte: Dados da Pesquisa. & & \\
\hline
\end{tabular}


sua ocorrência está o uso de dispositivos absorventes semelhantes a fraldas, cuja função é absorver o fluxo urinário( ${ }^{(21)}$.

A literatura científica aborda que indivíduos dependentes do uso de fraldas, devem realizar a troca diária no mínimo a cada seis horas. Isto é, em média quatro vezes durante o dia, independente da presença de eliminação, no intuito de evitar complicações ${ }^{(22)}$.

Nesse contexto, destaca-se que a execução de medidas preventivas de infecção relacionadas ao uso de fraldas, deve ser uma prática contínua adotada pelos enfermeiros atuantes em serviços de institucionalização de idosos.

Dentro do Componente Biológico/ Fisiológico, o DE "Marcha prejudicada" e "Risco de queda", apresentaram supremacia e prevaleceram em $94,4 \%$ dos idosos considerados frágeis.

Foi observado que os idosos tinham alterações na marcha e movimentos lentos. Um dos fatores determinantes de quedas em idosos é a presença de déficit de mar$\mathrm{cha}^{(23)}$. As pesquisas afirmam que a ocorrência de quedas na terceira idade são relacionadas em sua grande maioria a presença de fragilidade ${ }^{(24)}$.

Corroborando com esses achados, investigação realizada com idosos brasileiros, evidencidou relação estatisticamente significativa entre o risco para quedas e a síndrome da fragilidade ${ }^{(25)}$.

Diante dessa realidade, com o propósito de reduzir a ocorrência de quedas, o enfermeiro que atua nesse âmbito pode realizar palestras educativas conscientizando os idosos e demonstrando como evitar esse agravo, como também, faz-se necessário identificar os grupos em situação de risco ${ }^{(26)}$.

Além disso, é importante que o enfermeiro preste auxílio aos idosos frágeis que apresentam prejuízos na marcha. Investigação recente, sugeriu que as ILPIs devem ofertar dispositivos que auxiliem à deambulação dos idosos, quando necessário ${ }^{(27)}$.

No que se refere ao Componente Psicológico, o DE "Memória prejudicada" esteve presente na maioria dos idosos frágeis, mas nenhum apresentou diagnóstico sugestivo para demência durante a avaliação cognitiva.

No processo de envelhecimento é comum que algumas funções cognitivas,

Os enunciados de

\section{DE elaborados}

retratam situações

de vulnerabilidade

à saúde dos idosos

institucionalizados,

influenciadas por

fatores biológicos,

psicológicos e sociais.

A execução do estudo

evidenciou que $72 \%$

dos participantes

apresentaram

fragilidade, onde foi

possível identificar 8

DE entre os idosos

frágeis, possibilitando

a formulação de

intervenções frente

as necessidades de

cuidados. como a memória, diminuam. Portanto, o enfermeiro pode fazer o uso de técnicas de memória e sempre se manter atento a queixas de esquecimento, que podem predizer o risco de demência ${ }^{(28)}$.

Quanto ao Componente Social, o DE "Humor deprimido" foi constatado em $50 \%$ dos idosos fragilizados. Segundo um estudo de meta-análise, os idosos frágeis são quatro vezes mais vulneráveis ao desenvolvimento de depressão, quando comparados aos que não apresentam fragilidade. No entanto, embora a associação entre fragilidade e depressão sejam consideradas bidirecionais, não apresentam causalidade bem definidas ${ }^{(29)}$. Mediante o exposto, o gerenciamento do humor pelo enfermeiro nesses idosos é um fator relevante.

O DE "Falta de apoio social", foi o menos acentuado entre os idosos frágeis, representando um percentual de apenas $11,1 \%$. Na institucionalização, a rede de apoio é crucial e capaz de constribuir na melhora do bem-estar e qualidade de vida dos idosos ${ }^{(30)}$. Por isso, é importante que o enfermeiro promova apoio social aos idosos com fragilidade.

\section{CONCLUSÃO}

Os enunciados de DE elaborados retratam situações de vulnerabilidade à saúde dos idosos institucionalizados, influenciadas por fatores biológicos, psicológicos e sociais. A execução do estudo evidenciou que $72 \%$ dos participantes apresentaram fragilidade, onde foi possível identificar 8 DE entre os idosos frágeis, possibilitando a formulação de intervenções frente as necessidades de cuidados.

Os resultados encontrados, irão constribuir para o fortalecimento da prática profissional, reforçando a importância da identificação de idosos frágeis nos ambientes institucionalizados. Tal fato demonstra a necessidade da realização do Processo de Enfermagem como parte primordial do cuidado ao idoso fragilizado, auxiliando o direcionamento das ações da assistência de enfermagem de forma sistematizada, individualizada e resolutiva. 


\section{artigo}

\section{REFERÊNCIAS}

1. Miranda GMD, Mendes ACG, Silva ALA. Envelhecimento populacional no Brasil: desafios e consequências sociais atuais e futuras. Rev. bras. geriatr. gerontol. 2016; 19 (3): 507-519.

2. Cozzolino ASM, Gatti AL, Salles RJ. Atividade, sentimentos e percepções de mulheres diante do processo de envelhecimento. Bol. - Acad. Paul. Psicol. 2019; 39(96): 25-32.

3. Oliveira JM, Rozendo CA. Instituição de longa permanência para idosos: um lugar de cuidado para quem não tem opção? Rev. bras. enferm. 2014; 67(5): 773-779.

4. Borges CL, Silva MJ, Clares JWB, Bessa MEP, Freitas MC. Avaliação da fragilidade de idosos institucionalizados. Acta paul. enferm. 2013; 26(4):318-322.

5. Andrade AN, Fernandes MGM, Nóbrega MML, Garcia TR, Costa KNFM. Análise do conceito fragilidade em idosos. Texto contexto enferm. 2012; 21(4): 748-756.

6. Souza DS, Berlese DB, Cunha GL, Cabral SM, Santos GA. Análise da relação do suporte social e da síndrome de fragilidade em idosos. Psic., Saúde \& Doenças. 2017; 18(2): 420-433.

7. Melo EMA, Marques APO, Leal MCC, Melo HMA. Síndrome da fragilidade e fatores associados em idosos residentes em instituições de longa permanência. Saúde em Debate. 2018; 42(117): 468-480.

8. Duarte YAO, Nunes DP, Andrade FB, Corona LP, Brito TRP, Santos JLF, et al. Fragilidade em idosos no município de São Paulo: prevalência e fatores associados. Rev. bras. epidemiol. 2018; 21(Suppl 2): e180021.

9. Conselho Federal de Enfermagem. Resolução n. 358, de 15 de outubro de 2009: dispõe sobre a Sistematização da Assistência de Enfermagem e a implementação do Processo de Enfermagem em ambientes, públicos ou privados, em que ocorre o cuidado profissional de enfermagem. Brasilia: COFEN; 2009

10. Medeiros ACT, Nóbrega MML, Rodrigues RAP, Fernandes MGM. Nursing diagnoses for the elderly using the International Classification for Nursing Practice and the activities of living model. Rev Latino-Am Enfermagem. 2013; 21(2):523-30.

11. Fernandes BKC, Clares JWB, Borges CL, Nóbrega MML, Freitas MC. Diagnósticos de enfermagem para idosos institucionalizados fundamentados na teoria de Henderson. Rev. esc. enferm. USP. 2019; 53: e03472.

12. Pinheiro FM, Santo FHE, Chibante CLP, Pestana LC. Perfil de idosos hospitalizados segundo viginia henderson: contribuições para o cuidado em enfermagem. Care Online. 2016; 8(3):4789-4795.

13. Bertolucci PHF, Brucki SMD, Campacci SR, Juliano Y. The Mini-MentalStateExamination in anoutpatientpopulation: influenceofliteracy. Arquivos de Neuro-Psiquiatria. 1994; 52(1): 1-7.

14. Fabrício-Wehbe SCC. Adaptação cultural e validação da "Edmonton Frail Escale" (EFS) escala de avaliação de fragilidade em idosos [tese]. Ribeirão Preto: Escola de Enfermagem de Ribeirão Preto da USP; 2008.

15. Garcia TR, Coenen AM, Bartz CC. Classificação internacional para a prática de enfermagem CIPE ${ }^{\circledR}$ : versão 2017. ed. Artmed; 2016.
16. Brasil. Ministério da Saúde. Resolução n 466 de dezembro de 2012. Comissão Nacional de Ética em Pesquisa. CONEP. 2012

17. Assumpção D, Borim FSA, Francisco PMSB, Neri AL. Fatores associados ao baixo peso em idosos comunitários de sete cidades brasileiras: Estudo FIBRA. Ciênc. saúde coletiva. 2018; 23(4): 11431150.

18. Malta MB, Papini SJ, Corrente JD. Avaliação da alimentação de idosos de município paulista: aplicação do Îndice de Alimentação Saudável. Ciênc. saúde coletiva. 2013; 18(2): 377-384.

19. Brito MMS. Prevalência de incontinência urinária e fecal e fatores associados no escopo da síndrome da fragilidade em idosos comunitários: estudo da rede fibra [dissertação]. Belo Horizonte: Escola de Educação Física, Fisioterapia e Terapia Ocupacional da UFMG; 2015.

20. Offermans MPW, Du Moulin MFMT, Hamers JPH, Dassen T, Halfens RJG. Prevalence of urinary incontinence and associated risk factors in nursing home residents: a systematic review. Neurourol Urodyn. 2009; 28(4): 288-94.

21. Melo LS, Ercole FF, Oliveira DU, Pinto TS, Victoriano MA, Alcoforado CLGC. Infecção do trato urinário: uma coorte de idosos com incontinência urinária. Rev. Bras. Enferm. 2017; 70(4): 838-844.

22. Bitencourt GR, Alves LAF, Santana RF. Prática do uso de fraldas em adultos e idosos hospitalizados: estudo transversal. Rev. Bras. Enferm. 2018; 71(2): 343-349.

23. Duarte GP, Santos JLF, Lebrão ML, Duarte YAO. Relação de quedas em idosos e os componentes de fragilidade. Rev. bras. Epidemiol. 2018; 21( Suppl 2): e180017.

24. Fhon JRS, Rosset I, Freitas CP, Silva AO, Santos JLF, Rodrigues RAP. Prevalência de quedas de idosos em situaçao de fragilidade. Rev. Saúde Pública. 2013; 47(2): 266-273.

25. Sousa, JAV, Chuertniek JA, Grden CRB, Barreto MFC, Reche PM. Síndrome da fragilidade e risco para quedas em idosos: um estudo descritivo. Online Brazilian Journal of Nursing. 2015; 14(4): 508514.

26. Neto AHA, Patrício ACFA, Ferreira MAM, Rodrigues BFL, Santos TD, Rodrigues TDB, et al. Falls in institutionalized older adults: risks, consequences and antecedents. Rev Bras Enferm. 2017; 70(4):719-25.

27. Ferreira LMBM, Ribeiro KMOB, Jerez-Roig J, Araújo JRT, Lima LC. Quedas recorrentes e fatores de risco em idosos institucionalizados. Ciênc. saúde coletiva. 2019; 24(1): 67-75.

28. Ribeiro IA, Lima LR, Volpe CRG, Funghetto SS, Rehem TCMSB, Stival MM. Síndrome do idoso frágil em idosos com doenças crônicas na Atenção Primária. Rev. esc. enferm. USP. 2019; 53: e03449.

29. Soysal P, Veronese N, Thompson T, Kahl KG, Fernandes BS, Prina $A M$, et al. Relationship between depression and frailty in older adults: a systematic review and meta-analysis. Ageing Res Rev. 2017; 36:78-87.

30. Rodrigues AG, Silva AA. A rede social e os tipos de apoio recebidos por idosos institucionalizados. Rev. bras. geriatr. gerontol. 2013; 16(1): 159-170. 